\title{
Results from LHCb in charmless baryonic $B$ meson decays
}

\author{
Eduardo Rodrigues ${ }^{* \dagger}$ \\ University of Cincinnati \\ E-mail: rodrigedeucmail.uc.edu
}

\begin{abstract}
A review of the latest analyses from the LHCb experiment on charmless baryonic $B$-meson decays is presented. Emphasis is given on the first observation of a baryonic decay of the $B_{s}^{0}$ meson, namely the decay mode $B_{s}^{0} \rightarrow p \bar{\Lambda} K^{-}$. Decays of $B$ mesons to final states containing baryons are now observed for all $B$-meson species.
\end{abstract}

9th International Workshop on the CKM Unitarity Triangle

28 November - 3 December 2016

Tata Institute for Fundamental Research (TIFR), Mumbai, India

*Speaker.

†n behalf of the LHCb collaboration. 


\section{Introduction}

The decays of $B$ mesons to final states containing baryons have unique characteristics not found in similar decays involving mesons only. For a start, the inclusive branching fraction for baryonic $B$ decays, is rather large, roughly $7.5 \%$ of the $B$ total width [1]. The inclusive measurement was obtained from a study of the branching fractions of $B$ mesons to the $p, \Lambda$ and $\Xi^{-}$baryons accounting for various correlations, yielding an approximately equal rate of protons and neutrons. The hierarchy of baryonic $B$ decays is unusual in that many-body final states tend to have a larger branching fraction than 3- and 2-body final states. Last but not least, most baryonic decays show a pronounced enhancement at threshold in the baryon-antibaryon invariant mass. The theoretical description is a challenge and experimental information is scarce, hence needed to provide input to the various phenomenology models available.

The latest studies from the LHCb experiment [2,3] on charmless baryonic $B$-meson decays are here presented. All analyses are based on a $p p$ collision data sample collected in 2011 and 2012 at centre-of-mass energies of 7 and $8 \mathrm{TeV}$, respectively, corresponding to a total integrated luminosity of $3 \mathrm{fb}^{-1}$. The inclusion of charge-conjugate processes is implied throughout these proceedings.

\section{First observation of a baryonic $B_{s}^{0}$ decay}

Baryonic $B$ decays have been observed for all species except the $B_{s}^{0}$ meson. Since twobody baryonic $B$ decays are suppressed with respect to decays to multi-body final states $[4,5]$, the three-body decay $B_{s}^{0} \rightarrow p \bar{\Lambda} K^{-}$is a good candidate for a search for baryonic $B_{s}^{0}$ decays. The branching fraction has been predicted to be of the order of $10^{-6}[6]$, the same as for its similar mode $B^{0} \rightarrow p \bar{\Lambda} \pi^{-}$. The current experimental situation on the family of $B_{(s)}^{0} \rightarrow p \bar{\Lambda} h^{-}$decays $(h=\pi, K)$ and related modes such as $B_{(s)}^{0} \rightarrow p \bar{\Sigma}^{0} h^{-}$, with $\Sigma^{0} \rightarrow \Lambda \gamma$, is rather poor: the $B^{0} \rightarrow p \bar{\Lambda} \pi^{-}$decay has been studied by the BaBar [7] and Belle [8] collaborations; the Belle collaboration also reported the $90 \%$ confidence level upper limits $\mathscr{B}\left(B^{0} \rightarrow p \bar{\Lambda} K^{-}\right)<8.2 \times 10^{-7}$ and $\mathscr{B}\left(B^{0} \rightarrow p \bar{\Sigma}^{0} \pi^{-}\right)<3.8 \times 10^{-6}[9]$.

The LHCb analysis measures the branching fraction of the signal decay $B_{s}^{0} \rightarrow p \bar{\Lambda} K^{-}$relative to that of the normalisation mode $B^{0} \rightarrow p \bar{\Lambda} \pi^{-}$[10]. The $\Lambda \rightarrow p \pi^{-}$decays are reconstructed in two different categories: the first consists of $\Lambda$ hadrons that decay early enough for the daughter particles to be reconstructed in the vertex detector, and the second contains those that decay later such that track segments cannot be reconstructed in the vertex detector. These categories are referred to as long and downstream, respectively. Both $B_{s}^{0} \rightarrow p \bar{\Lambda} K^{-}$and $B^{0} \rightarrow p \bar{\Lambda} \pi^{-}$candidates are selected in a similar way throught the selection chain. After the hardware and software stages of the trigger, multilayer perceptrons are utilised to effectively separate signals from combinatorial background. Particle identification requirements separate the candidates in either the $p \bar{\Lambda} \pi^{-}$or the $p \bar{\Lambda} K^{-}$spectra. Background can arise from misidentified decays to the other signal final state; from $b$-hadron decays where one or more decay products are misidentified, such as decays with $K_{\mathrm{S}}^{0}$ mesons misidentified as $\Lambda$ baryons; and partially reconstructed backgrounds in which one or more particles from the decay of the $b$ hadron are not associated with the signal candidate, such as decays with a $\Sigma^{0}$ baryon. Extensive background studies are performed with simulation samples. 
The yields of the signal and background candidates in the eight subsamples are determined using a simultaneous unbinned extended maximum likelihood fit. The eight subsamples correspond to the 2011 and 2012 data-taking periods, the two $\Lambda$ reconstruction categories, and the two $p \bar{\Lambda} \pi^{-}$ and $p \bar{\Lambda} K^{-}$final states.
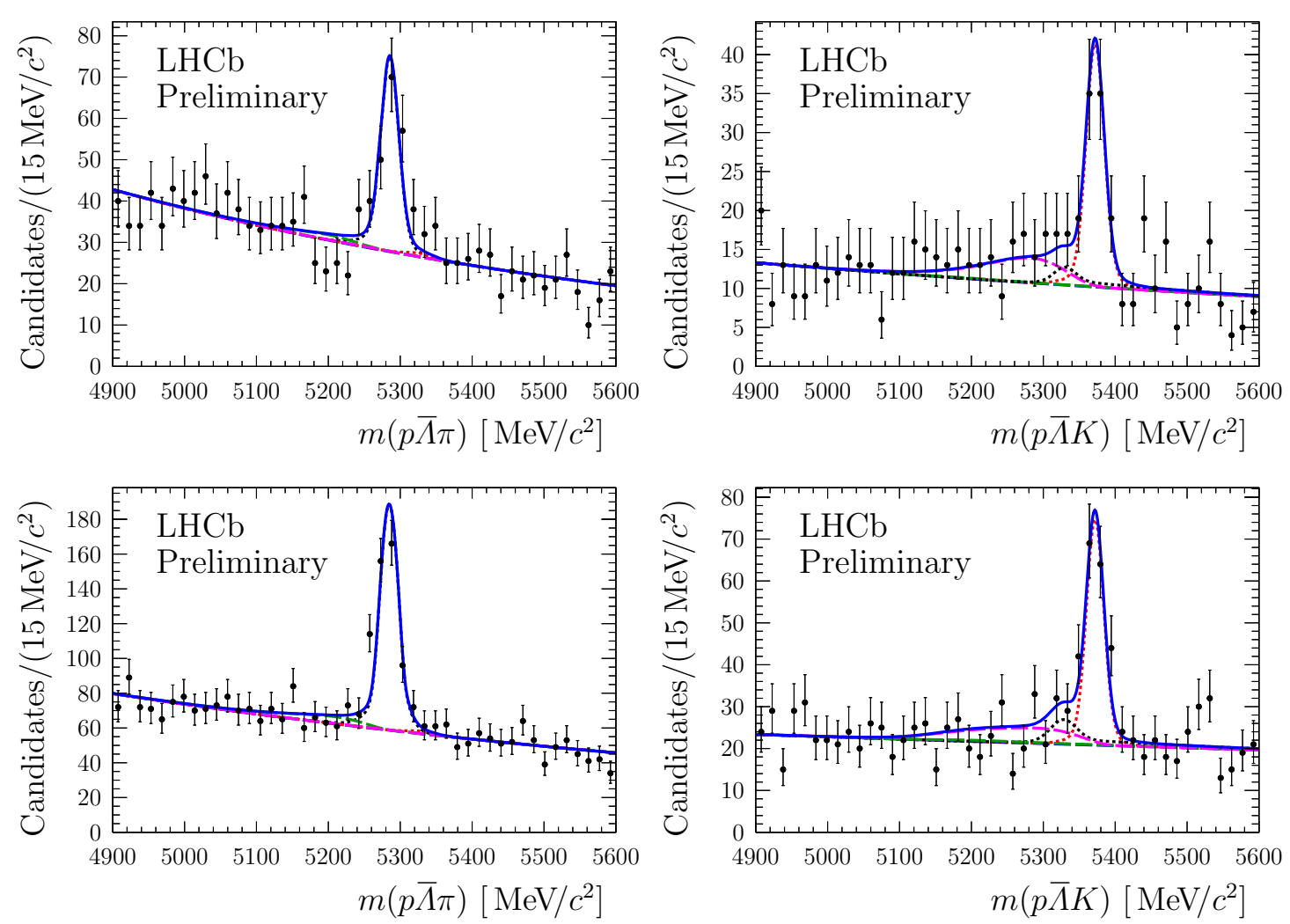

Figure 1: Results of the fit for the 2012 data sample reconstructed under (left) the $p \bar{\Lambda} \pi^{-}$invariant mass hypothesis and (right) the $p \bar{\Lambda} K^{-}$invariant mass hypothesis for (top) the long sample and (bottom) the downstream sample. The black points represent the data, the solid blue curve the complete fit model, the black (red) dotted curve the $B^{0} \rightarrow p \bar{\Lambda} \pi^{-}\left(B_{s}^{0} \rightarrow p \bar{\Lambda} K^{-}\right)$contribution, and the green (magenta) dashed curve the contribution from $B^{0} \rightarrow p \bar{\Sigma}^{0} \pi^{-}\left(B_{s}^{0} \rightarrow p \bar{\Sigma}^{0} K^{-}\right)$decays.

Figure 1 presents the fit to the $p \bar{\Lambda} h^{-}$invariant mass distributions for the 2012 subsamples. Prominent $B^{0} \rightarrow p \bar{\Lambda} \pi^{-}$and $B_{s}^{0} \rightarrow p \bar{\Lambda} K^{-}$signals are observed. The total signal yields $N\left(B^{0} \rightarrow\right.$ $\left.p \bar{\Lambda} \pi^{-}\right)=521 \pm 32$ and $N\left(B_{s}^{0} \rightarrow p \bar{\Lambda} K^{-}\right)=260 \pm 21$, where the uncertainties are statistical only. The $B_{s}^{0} \rightarrow p \bar{\Lambda} K^{-}$decay is observed with a statistical significance above 15 standard deviations, estimated from the change in log-likelihood between fits with and without the $B_{s}^{0} \rightarrow p \bar{\Lambda} K^{-}$signal component [11]. It is the first observation of a baryonic $B_{s}^{0}$ decay.

The distributions of the baryon-antibaryon system mass, $m(p \bar{\Lambda})$, are obtained with the $s$ Plot technique [12], see Fig. 2. They show the well-known pronounced enhancement at threshold first suggested in Ref. [13]. A threshold enhancement in baryonic $B_{s}^{0}$ decays is observed for the first time. 

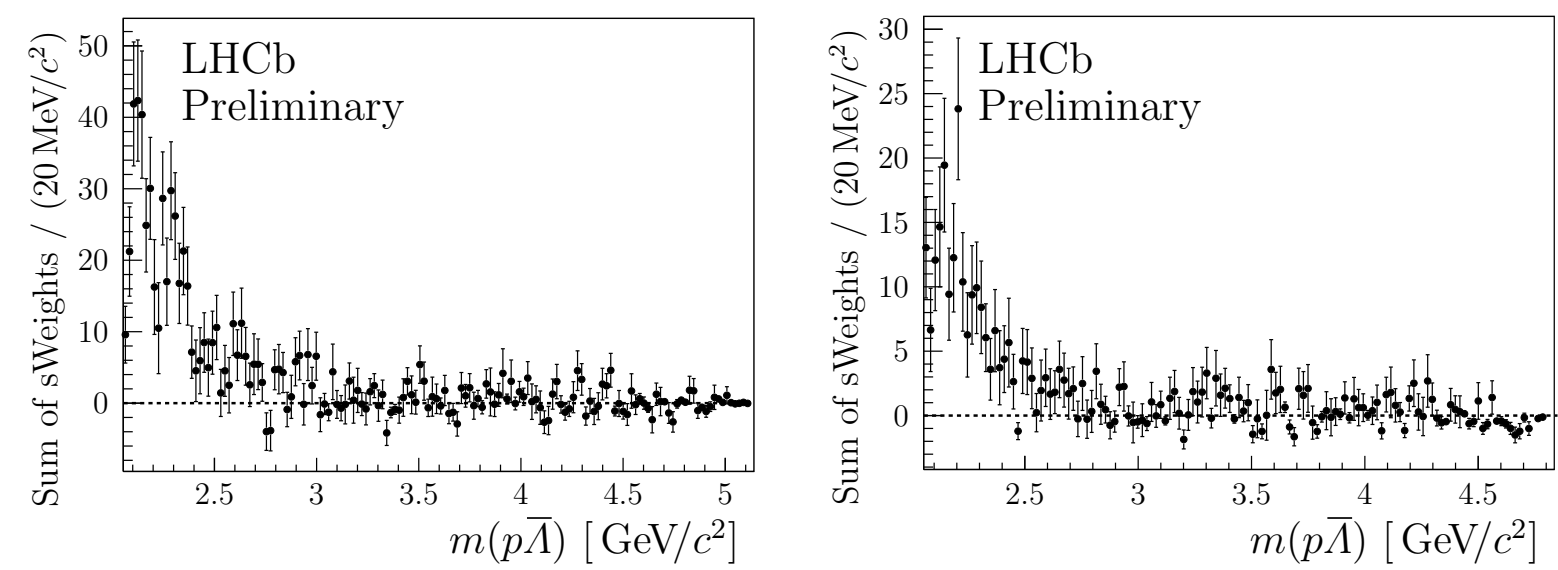

Figure 2: Invariant mass distributions of $m(p \bar{\Lambda})$ for (left) the $B^{0} \rightarrow p \bar{\Lambda} \pi^{-}$and (right) the $B_{s}^{0} \rightarrow$ $p \bar{\Lambda} K^{-}$decay mode. The background subtraction is performed using the sPlot technique. The distributions are not corrected for efficiency.

The branching fraction of the $B_{s}^{0} \rightarrow p \bar{\Lambda} K^{-}$decay is measured to be [10]

$$
\mathscr{B}\left(B_{s}^{0} \rightarrow p \bar{\Lambda} K^{-}+\bar{B}_{s}^{0} \rightarrow p \bar{\Lambda} K^{-}\right)=\left[5.48_{-0.80}^{+0.82} \pm 0.60 \pm 0.51(\mathscr{B}) \pm 0.32\left(f_{s} / f_{d}\right)\right] \times 10^{-6},
$$

where the first uncertainty is statistical and the second systematic, the third uncertainty accounts for the experimental uncertainty on the branching fraction of the $B^{0} \rightarrow p \bar{\Lambda} \pi^{-}$decay used for normalisation, and the fourth uncertainty relates to the knowledge of $f_{s} / f_{d}$. This branching fraction measurement is not corrected for a possible bias due to the finite width difference of the $B_{s}^{0}$ system [14]. The dominant source of systematic uncertainty comes from the poor knowledge of other baryonic $B$ decays representing backgrounds to the signals, which implies a challenging description of the spectra and non-negligible uncertainties on the fit model components and their characteristics.

Decays of $B$ mesons to final states containing baryons are now observed for all $B$-meson species. The results provide valuable information towards a better understanding of the dynamics of baryonic $B$ decays. In particular, further studies of the $B_{s}^{0}$ decays are welcome. So far, their phenonenology has focused on two-body final states $[4,15]$ and charmed baryonic decays [16].

\section{First evidence for $B^{+} \rightarrow p \bar{\Lambda}$}

The BaBar and Belle experiments have made numerous searches and observations of baryonic $B$ decays [17]. But only recently did the LHCb collaboration report the first observation of a twobody charmless baryonic $B^{+}$decay and the first evidence for a similar $B^{0}$ decay, namely $B^{+} \rightarrow$ $p \bar{\Lambda}(1520)$ [18] and $B^{0} \rightarrow p \bar{p}$ [19]. No other such type of decay has been observed, as $B$ decays to two-body baryonic final states are suppressed, with branching fractions typically one to two orders of magnitude lower than similar baryonic decays to multibody final states.

The decay $B^{+} \rightarrow p \bar{\Lambda}$ has been searched for by the CLEO [20] and Belle [21] collaborations. The most stringent experimental upper limit on its branching fraction is $3.2 \times 10^{-7}$ at $90 \%$ confidence level, determined by the Belle collaboration. The LHCb collaboration performed a search 
for this rare decay mode $B^{+} \rightarrow p \bar{\Lambda}$ using the topologically identical $B^{+} \rightarrow K_{\mathrm{S}}^{0} \pi^{+}$decay as normalisation channel in the determination of the branching fraction, to suppress common systematic uncertainties [22].

In the analysis, the candidates are selected in a similar way throught the selection chain for both the $B^{+} \rightarrow p \bar{\Lambda}$ signal decay and the normalisation channel $B^{+} \rightarrow K_{\mathrm{S}}^{0} \pi^{+}$. After the hardware and software stages of the trigger, multilayer perceptrons are utilised to effectively separate signals from backgrounds. Possible sources of non-combinatorial background to the $p \bar{\Lambda}$ and $K_{\mathrm{S}}^{0} \pi^{+}$spectra are investigated using simulation samples. The peaking background from $B^{+} \rightarrow p \bar{p} \pi^{+}$decays in the $p \bar{\Lambda}$ spectrum is found to be insignificant after selection. The currently unobserved $B^{+} \rightarrow p \bar{\Sigma}^{0}$ decay is treated as a source of systematic uncertainty.

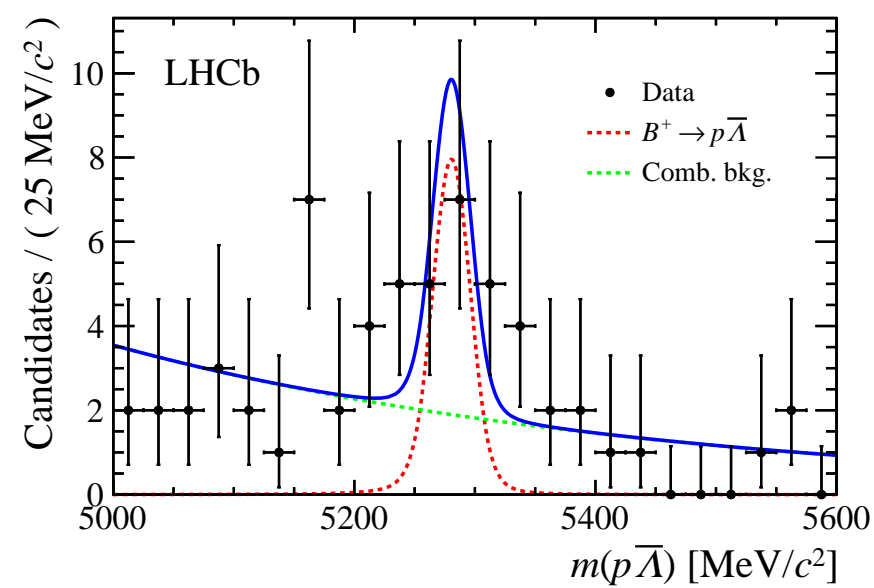

Figure 3: Invariant mass distribution of $p \bar{\Lambda}$ candidates after full selection. The result of the fit to the data (blue, solid) is shown together with each fit model component, namely the $B^{+} \rightarrow p \bar{\Lambda}$ signal and the combinatorial background.

The yields of the signal and background candidates in both the signal and normalisation samples are determined using unbinned extended maximum likelihood fits to the invariant mass spectra. The fit to the $p \bar{\Lambda}$ invariant mass distribution is presented in Fig. 3. An excess of $B^{+} \rightarrow p \bar{\Lambda}$ candidates with respect to background expectations is found, corresponding to a signal yield of $N\left(B^{+} \rightarrow p \bar{\Lambda}\right)=13.0_{-4.3}^{+5.1}$, where the uncertainties, obtained from a profile likelihood scan, are statistical only. The statistical significance of the $B^{+} \rightarrow p \bar{\Lambda}$ signal, of 4.1 standard deviations, is determined with a large set of samples simulated assuming the presence of background only. Inclusion of systematic uncertainties affecting the signal yield gives only a marginal change in the signal significance.

The $B^{+} \rightarrow p \bar{\Lambda}$ branching fraction is measured to be

$$
\mathscr{B}\left(B^{+} \rightarrow p \bar{\Lambda}\right)=\left(2.4_{-0.8}^{+1.0} \pm 0.3\right) \times 10^{-7},
$$

where the first uncertainty is statistical and the second systematic. The main sources of systematic uncertainty arise from the description of the fit model in the $p \bar{\Lambda}$ invariant mass and from uncertainties on the tracking efficiencies, which do not completely cancel given the nature of the final states of the signal and the normalisation channel. 
The measured branching fraction is compatible with the theoretical predictions in Refs. [15, 23] but is in tension with calculations based on QCD sum rules [24] and calculations based on factorisation with the hypothesis of the violation of partial conservation of the axial-vector current at the $\mathrm{GeV}$ scale [4].

\section{References}

[1] ARGUS, H. Albrecht et al., Measurement of inclusive B meson decays into baryons, Z. Phys. C42 (1989) 519.

[2] LHCb collaboration, A. A. Alves Jr. et al., The LHCb detector at the LHC, JINST 3 (2008) S08005.

[3] LHCb collaboration, R. Aaij et al., LHCb detector performance, Int. J. Mod. Phys. A30 (2015) 1530022, arXiv:1412.6352.

[4] Y. K. Hsiao and C. Q. Geng, Violation of partial conservation of the axial-vector current and two-body baryonic $B$ and $D_{s}$ decays, Phys. Rev. D91 (2015) 077501, arXiv:1407. 7639.

[5] H.-Y. Cheng and C.-K. Chua, On the smallness of tree-dominated charmless two-body baryonic $B$ decay rates, Phys. Rev. D91 (2015) 036003, arXiv: 1412.8272.

[6] C. Q. Geng, Y. K. Hsiao, and E. Rodrigues, Three-body charmless baryonic $\bar{B}_{s}^{0}$ decays, Phys. Lett. B 767 (2017) 205, arXiv:1612.08133.

[7] BaBar collaboration, B. Aubert et al., Measurement of the branching fraction and $\bar{\Lambda}$ polarization in $B^{0} \rightarrow \bar{\Lambda} p \pi^{-}$, Phys. Rev. D79 (2009) 112009, arXiv: 0904.4724.

[8] Belle collaboration, M.-Z. Wang et al., Study of $B^{+} \rightarrow p \bar{\Lambda} \gamma, p \bar{\Lambda} \pi^{0}$ and $B^{0} \rightarrow p \bar{\Lambda} \pi^{-}$, Phys. Rev. D76 (2007) 052004, arXiv:0 0404.2672.

[9] Belle collaboration, M. Z. Wang et al., Observation of $B^{0} \rightarrow p \bar{\Lambda} \pi^{-}$, Phys. Rev. Lett. 90 (2003) 201802, arXiv:hep-ex/0302024.

[10] LHCb collaboration, First observation of a baryonic Bo decay, LHCb-CONF-2016-016.

[11] S. S. Wilks, The large-sample distribution of the likelihood ratio for testing composite hypotheses, Ann. Math. Stat. 9 (1938) 60.

[12] M. Pivk and F. R. Le Diberder, sPlot: a statistical tool to unfold data distributions, Nucl. Instrum. Meth. A555 (2005) 356, arXiv: physics $/ 0402083$.

[13] W.-S. Hou and A. Soni, Pathways to rare baryonic B decays, Phys. Rev. Lett. 86 (2001) 4247, arXiv:hep-ph/0008079.

[14] K. De Bruyn et al., Branching ratio measurements of $B_{s}$ decays, Phys. Rev. D86 (2012) 014027, arXiv:1204.1735.

[15] C.-K. Chua, Charmless two-body baryonic $B_{u, d, s}$ decays revisited, Phys. Rev. D89 (2014) 056003, arXiv:1312.2335.

[16] Y. K. Hsiao and C. Q. Geng, $f_{J}(2220)$ and hadronic $\bar{B}_{s}^{0}$ decays, Eur. Phys. J. C75 (2015) 101, arXiv: 1412.4900 .

[17] BaBar and Belle collaborations, A. J. Bevan et al., The physics of the B factories, Eur. Phys. J. C74 (2014) 3026, arXiv:1406.6311.

[18] LHCb collaboration, R. Aaij et al., Evidence for CP violation in $B^{+} \rightarrow p \bar{p} K^{+}$decays, Phys. Rev. Lett. 113 (2014) 141801, arXiv:1407. 5907. 
[19] LHCb collaboration, R. Aaij et al., First evidence for the two-body charmless baryonic decay $B^{0} \rightarrow p \bar{p}$, JHEP 10 (2013) 005, arXiv:1308.0961.

[20] CLEO collaboration, T. E. Coan et al., Search for exclusive rare baryonic decays of B mesons, Phys. Rev. D59 (1999) 111101, arXiv: hep-ex/9810043.

[21] Belle collaboration, Y.-T. Tsai et al., Search for $B^{0} \rightarrow p \bar{p}, \Lambda \bar{\Lambda}$ and $B^{+} \rightarrow p \bar{\Lambda}$ at Belle, Phys. Rev. D75 (2007) 111101, arXiv: hep-ex/0703048.

[22] LHCb collaboration, R. Aaij et al., Evidence for the two-body charmless baryonic decay $B^{+} \rightarrow p \bar{\Lambda}$, arXiv:1611.07805, submitted to JHEP.

[23] H.-Y. Cheng and K.-C. Yang, Charmless exclusive baryonic B decays, Phys. Rev. D66 (2002) 014020 , arXiv:hep-ph/0112245.

[24] V. L. Chernyak and I. R. Zhitnitsky, B-meson exclusive decays into baryons, Nucl. Phys. B345 (1990) 137. 